\title{
A VARIATIONAL APPROACH TO BOUND STATES IN QUANTUM FIELD THEORY
}

\author{
H. Mishra and S.P. Misra \\ Institute of Physics, Bhubaneswar-751005 India
}

\begin{abstract}
We consider here in a toy model an approach to bound state problem in a nonperturbative manner using equal time algebra for the interacting field operators. Potential is replaced by offshell bosonic quanta inside the bound state of nonrelativistic particles. The bosonic dressing is determined through energy minimisation, and mass renormalisation is carried out in a nonperturbative manner. Since the interaction is through a scalar field, it does not include spin effects. The model however nicely incorporates an intuitive picture of hadronic bound states in which the gluon fields dress the quarks providing the binding between them and also simulate the gluonic content of hadrons in deep inelastic collisions.
\end{abstract}

Typeset Using REVTEX 


\section{INTRODUCTION}

We have recently considered a nonperturbative variational method for structure problems where the basic inputs are equal time algebra for the interacting field operators and a variational ansatz as may be appropriate for the specific dynamical situation. This has been applied to vacuum problem in Gross Neveu model [1] and in quantum chromodynamics for finite temperature and baryon densities [2]. It has further been applied to chiral symmetry breaking [3] where a new insight for vacuum structure with low energy hadronic properties is gained [4] as well as for the ground state of symmetric nuclear matter [5] and neutron matter [6]. We shall extend the method here to the consideration of bound states.

We have earlier considered this in a formal way [7] and in nuclear physics for deuteron [8]. In the present paper we shall extend this method to include renormalisation effects in a nonperturbative manner while considering dressing of fermions, and then examine quantitatively the boson content of the bound state as well as the momentum carried by these offshell quanta.

We organise the paper as follows. In section 2 we consider the Hamiltonian of two fermions interacting through Yukawa coupling along with a counter term. We next obtain the energy of a single dressed fermion and identify a nonperturbative renormalisation procedure for the present approximation scheme. In section $\mathbf{3}$ we construct two fermion bound state with the corresponding dressing through scalar quanta and determine the same again through energy extremisation. We also derive an effective two body potential in this section in terms of a momentum dependent coupling constant. In section 4 we illustrate the formalism with some explicit calculations of the binding energy including renormalisation effects. We also estimate the boson content of the bound state as a result of dressing and the momentum fraction carried by these offshell bosons. We tabulate the results to illustrate the effects of strong coupling. In section $\mathbf{5}$ we discuss the results.

The present problem as a parallel of "scalar" quantum chromodynamics is meant to be an analogue for the consideration of hadrons. We have consciously chosen a relatively simple 
problem to illustrate the nonperturbative nature of the dynamics for strong couplings. The results seem to reflect features of gluon content of hadrons as observed in deep inelastic collsions at the level of spectroscopy.

\section{FERMION DRESSING}

We shall consider here the interacting fermions and hence as stated the fermion will always be dressed with off-shell scalar quanta. We shall consider here the same for a single fermion. We need this for separating renormalisation effects.

The Hamiltonian we shall consider is given by

$$
H=H_{F}+H_{I N T}+H_{R}+H_{C T},
$$

where, $H_{F}$, the fermion kinetic term is given as

$$
H_{F}=\int d \vec{z} c^{\dagger}(\vec{z}) \epsilon_{z} c(\vec{z}) .
$$

In what follows we shall take the fermions to be non-relativistic so that $\epsilon_{z}=M-\vec{\nabla}_{z}^{2} / 2 M$. The interaction Hamiltonian $H_{I N T}$ is given as

$$
H_{I N T}=g \int d \vec{z} c(\vec{z})^{\dagger} c(\vec{z}) \phi(\vec{z}) .
$$

Further the free scalar field Hamiltonian $H_{R}$ is given as

$$
H_{R}=\frac{1}{2} \int d \vec{z}\left(\dot{\phi}^{2}+(\vec{\nabla} \phi)^{2}+\mu^{2} \phi^{2}\right) .
$$

In the above $\mu$ is the scalar field mass. Finally $H_{C T}$ will be the counter term to be identified with mass renormalisation.

We shall now expand the field operators $\phi$ in terms of creation and annihilation operators as [1,9.10]

$$
\begin{aligned}
\phi(\vec{z}) & =\frac{1}{\sqrt{2 \omega_{z}}}\left(a(\vec{z})^{\dagger}+a(\vec{z})\right), \\
\dot{\phi}(\vec{z}) & =i \sqrt{\frac{\omega_{z}}{2}}\left(-a(\vec{z})+a(\vec{z})^{\dagger}\right) .
\end{aligned}
$$


In the above $\omega_{z}$ is a differentiation operator which e.g. for free fields is given by $\left(-\vec{\nabla}_{z}^{2}+\mu^{2}\right)^{1 / 2}$. Further, here the meson fields satisfy here the equal time quantum algebra

$$
[\phi(\vec{x}), \dot{\phi}(\vec{y})]=i \delta(\vec{x}-\vec{y})
$$

When we substitute the expansions for the field operators as in equations (2) we have the usual commutation relations for the operators $a$ and $a^{\dagger}$ as

$$
\left[a(\vec{x}), a(\vec{y})^{\dagger}\right]=\delta(\vec{x}-\vec{y})
$$

Similarly for the fermion fields we have the anti commutation relations given as

$$
\left\{c(\vec{x}), c(\vec{y})^{\dagger}\right\}=\delta(\vec{x}-\vec{y})
$$

With the quantum algebra for the operators as above, we next wish to define the "dressed" particles as follows. We thus define the "physical" single fermion state as

$$
\left|\vec{x}>=c^{p h y}(\vec{x})^{\dagger}\right| v a c>=c(\vec{x})^{\dagger} U(\vec{x}) \mid v a c>
$$

where the unitary operator $U(\vec{x})$ is given as

$$
U(\vec{x})=\exp \left(B(\vec{x})^{\dagger}-B(\vec{x})\right)
$$

with the operator $B^{\dagger}$ given as

$$
B^{\dagger}(\vec{x})=\int d \vec{z} f(\vec{x}-\vec{z}) a(\vec{z})^{\dagger}
$$

Clearly $c(\vec{x})^{\dagger} U(\vec{x}) \equiv c^{\text {phys }}(\vec{x})^{\dagger}$ is the creation operator with the fermions being dressed. Thus the "physical" fermion operator as we have defined above, contains the bare fermion along with a coherent cloud of scalar quanta. The distribution of these quanta is described through the function $f(\vec{x}-\vec{z})$ which shall be determined through energy extremisation. We further note that from equations (9) (10) and (11)

$$
\begin{aligned}
<\vec{x} \mid \vec{y}> & =<\operatorname{vac}\left|U(\vec{x})^{\dagger} c(\vec{x}) c(\vec{y})^{\dagger} U(\vec{y})\right| \text { vac }> \\
& =\delta(\vec{x}-\vec{y})
\end{aligned}
$$


since $U(\vec{x})$ is unitary and commutes with $c(\vec{x})$. Hence the physical single fermion state has the usual orthogonality relation.

We shall now evaluate the hamiltonian expectation value with respect to the single fermion state of equation (9) which after extremisation will determine the function $f$. Thus we have

$$
<\vec{x}|H| \vec{x}^{\prime}>=\delta\left(\vec{x}-\vec{x}^{\prime}\right) h(f)
$$

where

$$
h(f)=h_{F}+h_{R}+h_{I N T} .
$$

In the above $h_{F}, h_{R}$ and $h_{I N T}$ correspond to the expectation values of the hamiltonian $H_{F}$, $H_{R}$ and $H_{I N T}$ of equations (1) respectively and are given explicitly as

$$
\begin{gathered}
h_{F}=M+\frac{1}{2 M} \frac{1}{(2 \pi)^{3}} \int d \vec{k} k^{2} \tilde{f}(\vec{k})^{2}, \\
h_{R}=\frac{1}{(2 \pi)^{3}} \int d \vec{k} \tilde{f}(\vec{k})^{2} \omega(k),
\end{gathered}
$$

and

$$
h_{I N T}=\frac{g}{(2 \pi)^{3}} \int d \vec{k} \sqrt{\frac{2}{\omega(k)}} \tilde{f}(\vec{k}) .
$$

In the above $\tilde{f}$ is the Fourier transform of $f$ given through

$$
f(\vec{x})=\frac{1}{(2 \pi)^{3}} \int d \vec{k} \tilde{f}(\vec{k}) e^{i \vec{k} \cdot \vec{x}}
$$

Extremising $h$ with respect to $\tilde{f}$ yields the optimum $\tilde{f}$ as

$$
\tilde{f}(\vec{k})=-g \sqrt{\frac{2}{\omega(k)}}\left(\frac{k^{2}}{M}+2 \omega(k)\right)^{-1} .
$$

In the coordinate space the function $f(\vec{x})$ is given as using equation (11)

$$
f(\vec{x})=-g \sqrt{\frac{2}{\omega_{x}}}\left(-\frac{\vec{\nabla}_{x}^{2}}{M}+2 \omega_{x}\right)^{-1} .
$$


The field at a point $\vec{z}$ due to a coherent cloud of scalar quanta associated with a fermion at $\vec{x}$ can be calculated as

$$
<\vec{x}|\phi(\vec{z})| \vec{x}>=\sqrt{\frac{2}{\omega_{x}}} f(\vec{x}-\vec{z})=-g \frac{2}{\omega_{x}}\left(-\frac{\vec{\nabla}_{z}^{2}}{M}+2 \omega_{x}\right)^{-1} \delta(\vec{x}-\vec{z}) .
$$

Clearly in the limit of the fermion mass $M \rightarrow \infty$ equation (21) reduces to the classical Yukawa field solution given as

$$
\begin{aligned}
f(\vec{x}-\vec{z}) & =-\frac{g}{\omega_{x}^{2}} \delta(\vec{x}-\vec{z}) \\
& =-\frac{g}{4 \pi} e^{-\mu|\vec{x}-\vec{z}|}
\end{aligned}
$$

Substituing the the expressions for $\tilde{f}(\vec{k})$ in equations (10) we have

$$
\begin{gathered}
h_{F}=M+\frac{g^{2}}{2 \pi^{2} M} \int \frac{k^{4}}{\omega(k)\left(k^{2} / M+2 \omega(k)\right)^{2}} d k, \\
h_{R}=\frac{g^{2}}{\pi^{2}} \int \frac{k^{2}}{\left(k^{2} / M+2 \omega(k)\right)^{2}} d k,
\end{gathered}
$$

and

$$
h_{I N T}=-\frac{g^{2}}{\pi^{2}} \int \frac{k^{2}}{\omega(k)\left(k^{2} / M+2 \omega(k)\right)} d k .
$$

From the three contributions of equations $(23),(24)$ and (25) above yields the "self energy" correction as

$$
\Delta M=h_{F}+h_{I N T}+h_{R}-M=-\frac{g^{2}}{2 \pi^{2}} \int \frac{k^{2}}{\omega(k)\left(k^{2} / M+2 \omega(k)\right)} d k .
$$

Clearly the above is logarithmically divergent and hence needs to be evaluated through a regularisation. We have to renormalise the mass with a counter term in the Lagrangian/ Hamiltonian. Hence with $\Delta M$ as in equation (26), we shall now take the counter term of equation (1) as

$$
H_{C T}=-\Delta M \int c(\vec{x})^{\dagger} c(\vec{x}) d \vec{x}
$$

We note that we have for the single particle state in the above included interactions through meson dressing as in equations (9) and (10) with a specific form for the same 
as in equation (11). $\mid \vec{x}>$ is not a single particle eigenstate of the Hamiltonian, but an approximation of the same. Trial states with all possible $B^{\dagger}$ shall generate the eigenstate. $\tilde{f}(k)$ as determined in equation (19) gives an optimal approximation for the form given by equation (11).

With the identification of the mass renormalisation as above with a coherent dressing of the fermion we shall next consider the bound state of two dressed fermions.

\section{TWO FERMION BOUND STATE}

We shall define here the two fermion bound state of total momentum zero using the dressed fermions $c^{\text {phys }}$ of equation (9) as follows.

$$
\left|B(\overrightarrow{0})>=\frac{1}{(2 \pi)^{3 / 2}} \int u(\vec{x}-\vec{y}) c_{1}^{p h y s}(\vec{x})^{\dagger} c_{2}^{p h y s}(\vec{y})^{\dagger} d \vec{x} d \vec{y}\right| v a c>
$$

Here $c_{i}^{\text {phys }}{ }^{\dagger}(\vec{x})(i=1,2)$ has the same form as in equation (11). Since we are considering the bound state, the distribution of the scalar quanta around the fermions inside the bound state will be different from that for single particle states. Thus we shall take for the operator $B^{\dagger}(\vec{x})$ of equation $(11)$ as

$$
B^{\dagger}(\vec{x})=\int d \vec{z} f_{1}(\vec{x}-\vec{z}) a(\vec{z})^{\dagger}
$$

where, $f_{1}$ is the dressing function of scalar quanta for the "constituent" fermions of the bound state and will be determined, as before, through energy extreamisation. Further the function $u(\vec{x}-\vec{y})$ is the conventional two particle wave funtion normalised as

$$
\int|u(\vec{r})|^{2} d \vec{r}=1
$$

With two body wave function normalised as above, it is trivial to check that $\mid B(\overrightarrow{0})>$ of equation (28) is formally normalised as, with equation (12),

$$
<B(\overrightarrow{0}) \mid B(\overrightarrow{0})>=\delta(\overrightarrow{0})
$$


Now we shall consider the expectation value of the Hamiltonian of equations (1) along with the counter term as given in equation (27) with respect to the state as given in equation (28). For the same we first note that, using translational invariance, the energy expectation value of the bound state will be given as [10]

$$
h\left(f_{1}\right)=(2 \pi)^{3}<B(\overrightarrow{0})|\mathcal{H}(\vec{z})| B(\overrightarrow{0})>
$$

where, $\mathcal{H}$ is the Hamiltonian density corresponding to equations (1) and (27). Thus from the fermionic kinetic term the contribution to the energy functional is given as

$$
h_{F}\left(\tilde{f}_{1}\right)=(2 \pi)^{3}<B(\overrightarrow{0})\left|c_{i}(\vec{z})^{\dagger}\left(M-\frac{\vec{\nabla}_{z}^{2}}{2 M}\right) c_{i}(\vec{z})\right| B(\overrightarrow{0})>=2 M+2 \Delta M_{F 1}+T_{F},
$$

where, $T_{F}$ is the conventional kinetic term given as

$$
\begin{aligned}
T_{F} & =\int u(\vec{r})^{*}\left(-\frac{\vec{\nabla}_{r}^{2}}{M}\right) u(\vec{r}) d \vec{r}, \\
& =\frac{1}{(2 \pi)^{3}} \int \tilde{u}(\vec{q})^{*}\left(\frac{q^{2}}{M}\right) \tilde{u}(\vec{q}) d \vec{q} .
\end{aligned}
$$

Further, $\Delta M_{F 1}$ is the contribution from the scalar quanta dressing given as

$$
\Delta M_{F 1}=\frac{1}{(2 \pi)^{3}} \frac{1}{2 M} \int k^{2} \tilde{f}_{1}(\vec{k})^{2} d \vec{k}
$$

In the above $\tilde{f}_{1}(\vec{k})$ is the Fourior transform of $f_{1}$ as in equation (18). We note that this equation is the second term in equation (15) where the fact that the dressing may change when the fermion is a part of the bound state is included.

The contribution from the interaction term is given as

$$
h_{\text {int }}\left(f_{1}\right)=(2 \pi)^{3}<B(\overrightarrow{0})\left|g c^{\dagger}(\vec{z}) c(\vec{z}) \phi(\vec{z})\right| B(\overrightarrow{0})>=T_{I N T}+\Delta M_{I N T 1},
$$

where, parallel to equation (17)

$$
\Delta M_{I N T 1}=\frac{1}{(2 \pi)^{3}} \int \sqrt{\frac{2}{\omega(k)}} \tilde{f}_{1}(\vec{k}) d \vec{k} .
$$

$T_{I N T}$ in equation (36) is given as

$$
T_{I N T}\left(f_{1}\right)=\frac{4 g}{(2 \pi)^{3}} \int C(\vec{q}) \frac{\tilde{f}_{1}(\vec{q})}{\sqrt{2 \omega(q)}} d \vec{q},
$$


where $C(\vec{q})$ is related to the fermion wave function as

$$
C(\vec{q})=(2 \pi)^{-3} \int \tilde{u}(\vec{p}+\vec{q} / 2) \tilde{u}(\vec{p}-\vec{q} / 2) d \vec{p}
$$

We have taken in the above $\tilde{u}(\vec{k})$ as the Fourier transform of $u(\vec{r})$ defined as

$$
u(\vec{r})=\frac{1}{(2 \pi)^{3}} \int \tilde{u}(\vec{k}) e^{i \vec{k} \cdot \vec{r}} d \vec{k}
$$

Clearly, from the normalisation of the two body wave function $C(\overrightarrow{0})=1$. In a similar manner the contribution from $H_{R}$ is

$$
h_{R}=<B(\overrightarrow{0})\left|\dot{\phi}^{2}-\vec{\nabla} \phi^{2}+\mu^{2} \phi^{2}\right| B(\overrightarrow{0})>=T_{R}+2 \Delta M_{R_{1}},
$$

where, $T_{R}$ is given as

$$
T_{R}=2 \times(2 \pi)^{-3} \int \tilde{f}_{1}(\vec{q})^{2} C(\vec{q}) \omega(\vec{q}) d \vec{q}
$$

In the above $C(\vec{q})$ is as defined in equation (39) and $\Delta M_{R 1}$ is given as

$$
\Delta M_{R 1}=(2 \pi)^{-3} \int \tilde{f}_{1}(\vec{k})^{2} \omega(k) d \vec{k}
$$

Thus the energy functional now becomes

$$
h\left(\tilde{f}_{1}\right)=T_{F}+T_{I N T}+T_{R}+2 M+2\left(\Delta M_{1}-\Delta M\right),
$$

where,

$$
\Delta M_{1}=\Delta M_{F 1}+\Delta M_{I N T 1}+\Delta M_{R 1},
$$

and, $\Delta M$ term arises from the counter term as in equation (27). Extremising the energy funtional $h\left(\tilde{f}_{1}\right)$ with respect to the funtion $\tilde{f}_{1}$ yields the optimum $\tilde{f}_{1}$ as

$$
\tilde{f}_{1}(\vec{k})=-g \sqrt{\frac{2}{\omega(k)}} \times \frac{(1+C(k))}{2 C(k) \omega(k)+2 \omega(k)+k^{2} / M} .
$$

Substituting the above expression for $\tilde{f}_{1}(\vec{k})$ in the expression for energy functional yields e.g. $T_{I N T}$ and $T_{R}$ as 


$$
T_{I N T}=-\frac{4 g^{2}}{(2 \pi)^{3}} \int \frac{C(\vec{q})(1+C(\vec{q}))}{\omega(q)\left[\left(q^{2} / M+2 \omega(q)+2 C(\vec{q}) \omega(q)\right]\right.} d \vec{q},
$$

and,

$$
T_{R}=\frac{4 g^{2}}{(2 \pi)^{3}} \int C(\vec{q})\left[\frac{1+C(\vec{q})}{q^{2} / M+2 \omega(q)+2 C(\vec{q}) \omega(q)}\right]^{2} d \vec{q} .
$$

It can be seen that when $\tilde{f}(\vec{k})$ is substituted in $\Delta M_{1}$ as in equation (45) is also ultra violet divergent. However the quantity $\Delta M_{1}-\Delta M$ does not have any ultra violet divergence and infact is given as

$$
\begin{aligned}
\Delta \epsilon & =\left(\Delta M_{1}-\Delta M\right) \\
& =\frac{g^{2}}{(2 \pi)^{3}} \int \frac{\left(q^{2} C(\vec{q}) / M\right)^{2}}{\omega(q)\left(q^{2} / M+2 \omega(q)\right)\left(2 C(\vec{q}) \omega(q)+q^{2} / M+2 \omega(q)\right)^{2}} d \vec{q} .
\end{aligned}
$$

Thus the energy of the bound state is given as

$$
E=2 M+T_{F}+T_{I N T}+T_{R}+2 \Delta \epsilon .
$$

We note that through mass renormalisation we had in equation (49) the difference of two divergent expressions which is convergent. Here an additional contribution to binding energy arises due to the renormalisation effect. This effect occurs in a nontrivial manner through minimisation with respect to meson dressing.

A comment regarding renormalisation is needed. The present treatment subtracts the one particle divergence in two particle interactions through conventional counter terms. This is as per the philosophy of renormalisation but is not equivalent to perturbative renormalisation. It is also incomplete since we do not know that it is applicable for any general ansatz for the dressing of fermions.

As may be noted in the above the contributions $T_{I N T}, T_{R}$ and $\Delta \epsilon$ constitute the potential energy. From the same we shall identify the two body potential in the next subsection.

\section{A. Two body potential}

For the identification of the two body potential, we shall compare the usual potential energy with energy expectation value as calculated here. With a two body potential $V(\vec{r})$ 
the corresponding expression for the potential energy is given by

$$
\begin{aligned}
V & =\int u(\vec{r})^{*} v(\vec{r}) u(\vec{r}) d \vec{r}, \\
& =\frac{1}{(2 \pi)^{3}} \int C(\vec{q}) \tilde{v}(\vec{q}) d \vec{q},
\end{aligned}
$$

where $u(\vec{r})$ is the two body wave function. In the second step above we have written the same in the momentum space using equation (40). Further $\tilde{v}(\vec{q})$ is the Fourier transform of $v(\vec{r})$ defined through

$$
v(\vec{r})=\frac{1}{(2 \pi)^{3}} \int \tilde{v}(\vec{q}) e^{i \vec{k} \cdot \vec{r}} d \vec{q}
$$

From equation (50) for the total energy, we also note that subtracting the conventional kinetic energy $2 M+T_{F}$ from the the total energy will give the potential energy. We shall use this fact to define the two body potential. Thus, the potential energy here arises from $T_{I N T}, T_{R}$ and $\Delta \epsilon$ of equations (47), (48) (49) and, with equation (52) the effective potential $\tilde{v}_{\text {eff }}(\vec{q})$ gets defined through

$$
V=T_{I N T}+T_{R}+\Delta \epsilon=\frac{1}{(2 \pi)^{3}} \int C(\vec{q}) \tilde{v}_{e f f}(\vec{q}) d \vec{q}
$$

where, the two body effective potential $\tilde{v}_{\text {eff }}(\vec{q})$ is given as

$$
\begin{aligned}
\tilde{v}_{e f f}(\vec{q})= & -\frac{g^{2}}{\omega(q)^{2}}\left[\frac{4(1+C(\vec{q}))}{\left(q^{2} / M \omega(q)+2+2 C(\vec{q})\right.}-4\left\{\frac{(1+C(\vec{q}))}{q^{2} / M \omega(q)+2+2 C(\vec{q})}\right\}^{2}\right. \\
& \left.-\frac{C(\vec{q})\left(q^{2} / M \omega(q)\right)^{2}}{\left(q^{2} / M \omega(q)+2\right)\left(q^{2} / M \omega(q)+2+2 C(\vec{q})\right)^{2}}\right] .
\end{aligned}
$$

We may rewrite the above equation as

$$
\tilde{v}_{e f f}(\vec{q})=-\frac{g_{e f f}(\vec{q})^{2}}{\omega(q)^{2}}
$$

where we have introduced a momentum dependent effective coupling $g_{\text {eff }}(\vec{q})$ given as

$$
g_{\text {eff }}^{2}(\vec{q})=g^{2} \times F(\vec{q})
$$

where, 


$$
\begin{aligned}
F(\vec{q})= & {\left[\frac{4(1+C(\vec{q}))}{q^{2} / M \omega(q)+2+2 C(\vec{q})}-4\left\{\frac{(1+C(\vec{q}))}{q^{2} / M \omega(q)+2+2 C(\vec{q})}\right\}^{2}\right.} \\
& \left.-\frac{C(\vec{q})\left(q^{2} / M \omega(q)\right)^{2}}{\left(q^{2} / M \omega(q)+2\right)\left(q^{2} / M \omega(q)+2+2 C(\vec{q})\right)^{2}}\right] .
\end{aligned}
$$

The above equation may be seen as the parallel of running coupling constant. Clearly for soft processes i.e. $q \rightarrow 0$ we have $g_{\text {eff }} \rightarrow g$ and $\tilde{V}_{\text {eff }}(\vec{q})$ goes over to the perturbative value $g^{2} / \omega(q)^{2}$. Also, when $q$ increases, $g_{e f f}$ decreases to zero. Such an idea of state and momentum dependent effective coupling constant is purely of dynamical origin, and does not arise from any renormalisation group equation.

Some comments regarding the definition of the potential as above may be relevant. The potential as in equation (55) contains fermion wave function $\tilde{u}(\vec{q})$ through $C(\vec{q})$. The parameters of this function will get determined through extremisation of energy of equation (32). Then only $\tilde{V}_{\text {eff }}(\vec{q})$ will be known after those parameters are substituted in equation (55). The potential as defined above depends upon the bound states of fermions and the fermion masses. A contribution as above may be one of the reasons for the fermion mass dependence of the potential in the consideration of heavy quarkonium spectroscopy [10,11]. In the present picture of deriving the potential, it happens to be an inevitable consequence of the formalism with simultaneous minimisation over meson dressing and fermion wave function.

We could analytically do the extremisation of the energy functional here because the energy functional was quadratic in the function $\tilde{f}_{1}$. This permitted an exact solution of the problem illustrating clearly the physical conclusions. However, if there is a quartic term $\lambda \phi^{4}$ in the potential, or there is a cubic and a quartic term, this extremisation cannot be explicitly done. However, by choosing a suitable basis for the unknown functions, extremisation can be done to a desired degree of accuracy. This opens up a new frontier for the determination of effective potentials. 


\section{SOME SIMPLE ILLUSTRATIONS}

We shall here first obtain the spin indepedent central potential as varying with the bound state wave function. For this purpose we take the two body wave function in the harmonic oscillator wave function basis to examine some features of the present nonperturbative approach. We take two body fermion wave function as

$$
u(\vec{r})=\cos \alpha u_{1}(\vec{r})+\sin \alpha \cos \beta u_{2}(\vec{r})+\sin \beta \sin \alpha u_{3}(\vec{r})
$$

where,

$$
\begin{aligned}
& u_{1}(\vec{r})=\left(\frac{1}{\pi R^{2}}\right)^{3 / 4} \exp \left(-\frac{r^{2}}{2 R^{2}}\right) \\
& u_{2}(\vec{r})=\sqrt{\frac{3}{2}\left(\frac{1}{\pi R^{2}}\right)^{3 / 4}\left(1-\frac{2 r^{2}}{3 R^{2}}\right) \exp \left(-\frac{r^{2}}{2 R^{2}}\right)} \\
& u_{3}(\vec{r})=\sqrt{\frac{15}{8}} \cdot\left(\frac{1}{\pi R^{2}}\right)^{\frac{3}{4}}\left(1-\frac{4 r^{2}}{3 R^{2}}+4 \frac{r^{4}}{15 R^{4}}\right) \exp \left(-\frac{r^{2}}{2 R^{2}}\right) .
\end{aligned}
$$

Clearly in the above we have taken the three terms of the basis and we shall see that it is sufficient to illustrate the results. With $u(\vec{r})$ as above $C(\vec{q})$ of equation (39) becomes

$$
C(\vec{q})=\sum a_{i j} C_{i j}(\vec{q})
$$

where, $i, j=1,3$ and

$$
C_{i j}(\vec{q})=(2 \pi)^{-3} \int \tilde{u}_{i}(\vec{p}+\vec{q} / 2) \tilde{u}_{j}(\vec{p}-\vec{q} / 2) d \vec{p}
$$

We may note that the two matrices $a$ and $C$ are symmetric in their indices with the different elements given as

$$
\begin{array}{ll}
a_{11}=\cos ^{2} \alpha, & a_{12}=\cos \alpha \sin \alpha \cos \beta, a_{22}=\sin ^{2} \alpha \cos ^{2} \beta \\
a_{13}=\cos \alpha \sin \beta \sin \alpha, a_{23}=\sin ^{2} \alpha \sin \beta \cos \beta, a_{33}=\sin ^{2} \alpha \sin ^{2} \beta
\end{array}
$$

and,

$$
C_{11}(\vec{q})=\exp \left(-\frac{q^{2} R^{2}}{4}\right)
$$




$$
\begin{aligned}
& C_{12}(\vec{q})=\sqrt{\frac{3}{2}} \cdot \frac{1}{6} q^{2} R^{2} \exp \left(-\frac{q^{2} R^{2}}{4}\right), \\
& C_{22}(\vec{q})=\left(1-\frac{1}{3} q^{2} R^{2}+\frac{1}{36} q^{4} R^{4}\right) \exp \left(-\frac{q^{2} R^{2}}{4}\right), \\
& C_{13}(\vec{q})=\sqrt{\frac{15}{8}} \cdot \frac{1}{60} \cdot q^{4} R^{4} \exp \left(-\frac{q^{2} R^{2}}{4}\right), \\
& C_{23}(\vec{q})=\frac{3 \sqrt{5}}{4}\left(\frac{2}{9} q^{2} R^{2}-\frac{2}{45} q^{4} R^{4}+\frac{1}{360} q^{6} R^{6}\right) \exp \left(-\frac{q^{2} R^{2}}{4}\right), \\
& C_{33}(\vec{q})=\left(1-\frac{2}{3} q^{2} R^{2}+\frac{11}{60} q^{4} R^{4}-\frac{1}{60} q^{6} R^{6}+\frac{1}{1920} q^{8} R^{8}\right) \exp \left(-\frac{q^{2} R^{2}}{4}\right) .
\end{aligned}
$$

With $C(\vec{q})$ as in equation (61), $T_{I N T}, T_{R}$ and $\Delta \epsilon$ as given in equations (47), (48) and (49) now become functions of the fermion wave funtion parameters $\alpha, \beta$ and $R$. Further the fermion kinetic term $T_{F}$ now becomes

$$
T_{F}=\sum a_{i j} T_{i j}
$$

with,

$$
\begin{gathered}
T_{11}=\frac{3}{2} \frac{1}{M R^{2}} ; \quad T_{12}=\sqrt{\frac{3}{2}} \frac{1}{M R^{2}} ; \quad T_{22}=\frac{7}{2} \frac{1}{M R^{2}} ; \\
T_{13}=0 ; \quad T_{23}=\sqrt{5} \cdot \frac{1}{M R^{2}} ; \quad T_{33}=\frac{11}{2} \cdot \frac{1}{M R^{2}} .
\end{gathered}
$$

We next extremise the energy as given in equation (50) with respsct to the fermion wave funtion parameters $\alpha, \beta$ and $R$ for different couplings and for different values of scalar field mass, which yields the ground state energy. Once the parameters for the bound state are fixed through extremisation, we may calculate the number of scalar quanta in the bound state. This is given as

$$
N=<B(\overrightarrow{0})\left|a^{\dagger}(\vec{z}) a(\vec{z})\right| B(\overrightarrow{0})>=2 \times(2 \pi)^{-3} \int \tilde{f}_{1}(\vec{q})^{2} C(\vec{q}) d \vec{q} .
$$

As a post facto check for the nonrelativistic approximation for the fermions one may calculate the average momentum of the fermions inside the bound state which is given as

$$
\frac{<P_{F}^{2}>}{M^{2}}=\frac{1}{(2 \pi)^{3}} \int \tilde{u}(\vec{q})^{*}\left(\frac{q^{2}}{M^{2}}\right) \tilde{u}(\vec{q}) d \vec{q} .
$$

As may be noted from table I, this quantity increases as the coupling is increased and illustrating how the nonrelativistic approximation for the fermions in the bound state ceases 
to be valid. We may include relativistic correction formally by replacing the kinetic term contribution $q^{2} / M$ in equations (34) and (35) by $2\left(\left(q^{2}+M^{2}\right)^{1 / 2}-M\right)$ as well as in the solution for $\tilde{f}_{1}(k)$ in equation (46). This replacement is not exact, but it enables us to use the solution of equation (46) of the nonrelativistic limit to make a qualitative extrapolation.

We next calculate the momentum carried by the constituent scalar quanta is given by

$$
<P_{S}^{2}>=<B(\overrightarrow{0})\left|\vec{\nabla} a^{\dagger}(\vec{z}) \vec{\nabla} a(\vec{z})\right| B(\overrightarrow{0})>=2 \times(2 \pi)^{-3} \int \tilde{f}_{1}(\vec{q})^{2} q^{2} C(\vec{q}) d \vec{q}
$$

The results of the above calculations are summerised in Table I.

We note that the renormalisation corrections as calculated here are consistently small. This is may be because we have constructed the bound states with dressed fermions so that the main effect of renormalisation has gone to define the physical mass. We also observe the nice result that the deviations of the energy expectation values from the perturbative expressions is not large. However, the distinction of the present method as opposed to that of the potentials shows clearly in momentum distributions. We see that as the coupling increases from 0.1 to 1 , the relative momentum fraction carried by the off-shell bosons increases from about $10 \%$ to $67 \%$. This clearly illustrates why gluons can carry about half the proton momentum in deep inelastic lepton proton collisions. Such a result arises here through the structure of the bound state. We further note that in the last column the average number of boson quanta increases from $6 \%$ when the coupling is 0.1 to as high as 288 for $g^{2} / 4 \pi=1$ and 3730 for $g^{2} / 4 \pi=1.5$ when we take $\mu=0$. If we take the mass of the boson quanta to be $0.01 \mathrm{M}$ the average number of these quanta rises from $3.5 \times 10^{-5}$ for coupling 0.1 to 1 and 3.4 for couplings 1 and 1.5 respectively. The difference in these numbers only reflects the presence of soft quanta for zero mass, and, illustrate that explicit gluon (or meson) dressing should be relevant for properties of hadrons (or nucleus).

This picture for spectroscopy has specific extra predictions for experimental observations. When we consider a probe which interacts only with the fermions, the presence of off-shell bosonic quanta simulating the potential will be known through momentum imbalance, as seen in deep inelastic collisions. If the probe interacts with boson quanta, they may also 
be directly "seen" through these interactions with the off-shell bosonic quanta of the bound state. This could be operative for hadron hadron collisions with the interaction of the gluons in hadrons. In making the above statements we are obviously extrapolating scalar mesons to that of gluons as a parallel for hadrons in a realistic environment, or to pions in case of the nucleus [8].

Further, as the scalar field mass increases, the coupling to form the bound state needs to be larger. For very strong couplings $\left(g^{2} / 4 \pi>2\right)$ we do not obtain a solution for the bound state; the range of Yukawa interactions here becoming much too small.

We end our illustration with a possible example for Higgs particles. We consider the case of heavy fermion bound state with Yukawa interaction where the coupling becomes proportional to mass of the fermion as in grand unification models [12]. Here we may have e.g. $g^{2} / 4 \pi=\sqrt{2} G_{F} M^{2} / 4 \pi$ with $G_{F}=1.7 \times 10^{-5} \mathrm{GeV}^{-2}$, being Fermi coupling constant as in the minimal Higgs model [12]. The results here are summarised in table II and the results are mostly similar to the results of Table I. The difference in this case, however, is that the momenta fraction carried by the off-shell Higgs quanta is of the same order as that of fermions even when the average number of meson quanta is quite small.

\section{DISCUSSIONS}

We would like to mention that the present paper is a part of a new approximation scheme through a variational method in field theory to vacuum structures or to bound states. For the later, the scheme consists of defining dressed single particle states [8] and then defining two particle bound states through further modifications in dressing. Only equal time algebra has been utilised. The energy of the one particle or of the bound system is calculated through an extremisation procedure. Since dressing is done through ansatz functions, a better approximation will consist of taking more general functions.

The present paper shows that the effects of such ansatz functions occur through highly

nonlinear expressions which were not observed in earlier formal analysis [7]. As expected, the 
one particle state contains the familiar divergence of self energy and needs renormalisation. The use of renormalisation here follows the familiar pattern of defining a counter term for a single particle state so that the energy of the single particle at rest is identified with its physical mass. We show that this counter term is adequate not to have any infinity when the two particle bound state is defined. Also, the counter term gives rise to a finite and state dependant additional contribution to the energy of the bound state as expected for any renormalisation procedure.

The prescription for renormalisation is still incomplete since we do not show here that for all ansatz functions the divergence disappears. We believe that if infinities survive for physically measurable quantities, the solution is likely to be inconsistent so that the ansatz function is not acceptable, but the proof of the same shall be nontrivial. Further we should probably include a $\lambda \phi^{4}$ term as a prototype of QCD to simulate the quartic couplings of gluons. Then we may also have bilinears in $\phi$ while considering dressing [2, 6, 13, 15]. However, when these are included, the problem becomes prohibitively complicated. In the present paper therefore we have implemented dressing to examine renormalisation for single particle states so as to discuss bound state spectroscopy related to other details of dynamics.

This picture of including the scalar quanta in the bound state as off-shell constituents instead of using a propagator or potential is not only aesthetically appealing but has phenomenological consequnces for strong interactions as considered in the last section. Also, the present formulation generates state and fermion mass dependant potential as needed in quarkonium spectroscopy, and, gives an insight in a natural manner why gluons carry about half the hadron momentum in deep inelastic collisions. We can thus ask and answer such questions while dealing with spectroscopy, and, although really a toy model, as analysed in the last section the results are close to the expected physical situation. 


\section{ACKNOWLEDGMENTS}

The authors are thankful to A. Mishra, S.N. Nayak and P.K. Panda many useful discussions. SPM would like to thank Department of Science and Technology, Government of India for the research grant no. SP/S2/K-45/89 for financial assistance. 


\section{REFERENCES}

[1] H. Mishra, S.P. Misra and A. Mishra, Int. J. Mod. Phys. A3, 2331 (1988).

[2] A. Mishra, H. Mishra, S.P. Misra and S.N. Nayak, Pramana (Jou. of Phys) 37, 59 (1991); ibid Z. Phys. C57, 233 (1993). A. Mishra, H. Mishra and S. P. Misra, Z. Phys. C (To appear).

[3] A. Mishra, H. Mishra, S.P. Misra and S.N. Nayak, Z. Phys. C57, 233 (1993); H. Mishra and S. P. Misra IP/BBSR/92-26.

[4] A. Mishra and S.P. Misra, Z. Phys. C58,325 (1993).

[5] A. Mishra, H. Mishra, and S.P. Misra Int. J. Mod. Phys. A5, 3391 (1990); H. Mishra, S.P. Misra, P.K. Panda and B.K. Parida, Int. J. Mod. Phys. E1, 405 (1992).

[6] H. Mishra, S.P. Misra, P.K. Panda and B.K. Parida, Int. J. Mod. Phys. E (To appear).

[7] S.P. Misra, Phys. Rev. D35, 2607 (1987), S.P. Misra, Ind. J. Phys. 61B, 287 (1987).

[8] S.P. Misra, P.K. Panda and R. Sahu, Phys. Rev. C45, 2079 (1992).

[9] D. Schutte, Phys. Rev. D31, 810 (1985).

[10] S.P. Misra, S. Naik and A.R. Panda, Pramana (J. Phys.) 28, 131 (1987).

[11] D.B. Lichtenberg, E. Predenzzi, R. Roncaglia, M. Rosso and J.G. Wills, Z. Phys. C41, $615(1989)$.

[12] H. Inazawa and T. Morri, Phys. Lett. B203, 279 (1988).

[13] P. M. Stevenson, Phys. Rev. D 32, 1389 (1985);

[14] P. M. Stevenson, G.A. Hajj and J.F. Reed, Phys. Rev. D34,

[15] H. Mishra and A. R. Panda, J. Phys. G (Nucl. Part. Phys.) 18, 1301 (1992). 


\section{TABLES}

TABLE I.

\begin{tabular}{|c|c|c|c|c|c|c|}
\hline$\frac{g^{2}}{4 \pi}$ & $E-2 M$ & $2 \times \Delta \epsilon$ & $E_{\text {nonpert }} / E_{\text {pert }}$ & $\frac{\left\langle P_{F}^{2}\right\rangle}{M^{2}}$ & $\frac{\left\langle P_{S}^{2}\right\rangle}{M^{2}}$ & Avg. no. of scalar quanta \\
\hline \multicolumn{7}{|c|}{ Scalar field mass $\mu=0$} \\
\hline 0.01 & $-2.3 \times 10^{-5}$ & $1.7 \times 10^{-11}$ & 1.00 & $2.4 \times 10^{-5}$ & $2.3 \times 10^{-7}$ & $1.5 \times 10^{-4}$ \\
\hline 0.1 & $-2.3 \times 10^{-3}$ & $4.2 \times 10^{-7}$ & 1.00 & $2.4 \times 10^{-3}$ & $2.1 \times 10^{-4}$ & $6.1 \times 10^{-2}$ \\
\hline 1.0 & -.244 & $3.6 \times 10^{-3}$ & 0.92 & 0.31 & 0.20 & 288 \\
\hline 1.5 & -0.618 & 0.02 & 0.81 & 1.33 & 1.02 & 3730 \\
\hline \multicolumn{7}{|c|}{ Scalar field mass $\mu=0.01 M$} \\
\hline 0.1 & $-1.5 \times 10^{-3}$ & $5.2 \times 10^{-7}$ & 1 & $2.2 \times 10^{-3}$ & $1.9 \times 10^{-4}$ & $3.5 \times 10^{-5}$ \\
\hline 1.0 & -0.24 & $3.8 \times 10^{-3}$ & 0.92 & 0.32 & 0.2 & 1.01 \\
\hline 1.5 & -0.61 & 0.02 & 0.81 & 1.32 & 1.02 & 3.4 \\
\hline
\end{tabular}

TABLE II.

\begin{tabular}{|c|c|c|c|c|c|c|c|}
\hline $\mathrm{M}(\mathrm{GeV})$ & coupling & $(\mathrm{E}-2 \mathrm{M})(\mathrm{Gev})$ & $2 \times \Delta \epsilon(\mathrm{GeV})$ & $E_{\text {nonpert }} / E_{\text {pert }}$ & $\frac{\left\langle P_{F}^{2}\right\rangle}{M^{2}}$ & $\frac{\left\langle P_{S}^{2}\right\rangle}{M^{2}}$ & Avg.No. of scalar quanta \\
\hline \multicolumn{8}{|c|}{$\mu=10 \mathrm{GeV}$} \\
\hline 400 & 0.21 & -2.33 & $3.5 \times 10^{-3}$ & 0.99 & $9.63 \times 10^{-3}$ & $9.56 \times 10^{-3}$ & $1.33 \times 10^{-3}$ \\
\hline 600 & 0.47 & -26.9 & 0.13 & 0.97 & $5.5 \times 10^{-2}$ & $5.31 \times 10^{-2}$ & 0.132 \\
\hline 800 & 0.84 & -128.2 & 1.5 & .937 & 0.206 & 0.188 & 0.607 \\
\hline 1000 & 1.32 & -440.32 & 12.0 & 0.895 & 0.777 & 0.603 & 2.21 \\
\hline \multicolumn{8}{|c|}{$\mu=100 \mathrm{GeV}$} \\
\hline 600 & 0.47 & -2.38 & 0.03 & 0.92 & $2.85 \times 10^{-2}$ & $2.80 \times 10^{-2}$ & $1.3 \times 10^{-2}$ \\
\hline 800 & 0.84 & -67.3 & 1.2 & 0.905 & 0.183 & 0.168 & 0.184 \\
\hline 1000 & 1.32 & -333.1 & 11.3 & 0.828 & 0.754 & 0.588 & 0.938 \\
\hline
\end{tabular}

